\title{
A COMPUTATIONAL ANALYSIS OF BLOOD FLOW THROUGH PORTAL VEIN UNDER NORMAL AND EXTRAHEPATIC OBSTRUCTIONS
}

\author{
MST. KHORSEDA AKTAR* AND MD. TAJUL ISLAM ${ }^{1}$ \\ Department of Mathematics, Begum Rokeya University, Rangpur, Bangladesh
}

\begin{abstract}
Portal hypertension is an alarming disease globally whereas Extrahepatic portal vein obstruction (EHPVO) is an important cause of portal hypertension among children in most of the Asian countries. The frequent occurrence of this disease is due to the formation of thrombosis in the portal vein. To acquire insights into the behaviour of hemodynamics, EHPVO case is studied by the technique of computational fluid dynamics (CFD) by considering partial block formation with different sizes inside the main portal vein. On the basis of block sizes, three cases namely normal portal vein (without block in the portal vein) case, $40 \%$ block in the portal vein case and $85 \%$ block in the portal vein case are simulated by assuming a 2D steady, laminar and fully developed flow with no-slip wall condition using Ansys ${ }^{\mathrm{TM}}$ Fluent. Our study reveals that the flow distribution between the left and right branches of the portal vein is significantly influenced by the formation of the block in the main portal vein. It is concluded that Extrahepatic portal vein obstruction leads to the possibility of Hypertrophy and Atrophy complex to the liver and could heighten the risk of liver diseases. Our numerical examination will play a great role to the Liver Specialist for understanding the flow behavior with obstructed flow path as well as the diagnosis of associated liver disease and their treatments.
\end{abstract}

Key words: Portal hypertension, Extrahepatic portal vein obstruction, Computational Fluid dynamics, Hemodynamic, Hypertrophy, Atrophy

\section{INTRODUCTION}

The portal vein (PV) is formed by the confluence of the splenic vein and the superior mesenteric vein behind the neck of the pancreas. The portal vein is responsible for around two thirds of the blood flow that rich in oxygen, nutrients growth factors, hormones, among other elements. In the hilum of the liver, a portal vein divides into the left and right portal vein branches which supply blood to the left and right sides of the liver respectively.

Portal hypertension $(\mathrm{PH})$ is caused by altered hemodynamics in the liver. One alteration is increased resistance to flow of blood through the liver and a second is a large increase in the amount of blood trying to enter the liver from the splanchnic circulation

\footnotetext{
* Corresponding author:<keyecard@gmail.com>.

${ }^{1}$ Department of Mathematics, Begum Rokeya University, Rangpur, Bangladesh
} 
(Sherlock and Dooley 2002). PH is used to describe elevated pressures in the portal venous system. The elevated pressure is characterized by a gradient of greater than 10 $\mathrm{mm} \mathrm{Hg}$ between the portal venous and central venous pressures (Bari et al. 2012). Causes of increased resistance of blood flow into the liver can be presinusoidal, intrasinusoidal, or postsinusoidal. Presinusoidal causes include blockage of the main portal vein or annular fibrosis of intrahepatic portal venules. Intrasinusoidal causes are mainly due to cirrhosis and include subendothelial deposition of collagen in the space of disse, distortion from regenerating nodules, constriction due to synthesis of NO and increased vasocontrictors (endothelin), and impaired hepatic removal and increased consumption of endotoxins or compression by tumors. Postsinusoidal causes include veno-occlusive disease, obstruction of small hepatic venules, Budd Chiari Syndrome, obstruction of the main hepatic vein, and severe right side congestive heart failure (see Sherlock and Dooley 2002) and Sherlock (1974) classified, an older classification, PH into two main groups - presinusoidal and intrahepatic. The presinusoidal is further divided into extrahepatic, in which the obstruction is in the main portal vein. Portal hypertension is one of the major complications in patients with diseases of the liver, such as liver cirrhosis, veno-occlusive disease, idiopathic extrahepatic portal vein obstruction and prehepatic portal idiopathic pathology (Denk 2004, British Liver Trust).

Portal vein thrombosis (PVT) is an important cause of portal hypertension. PVT refers to thrombosis that develops in the trunk of the portal vein or including its right and left intrahepatic branches and may even extend to the splenic or superior mesenteric veins or towards the liver involving intrahepatic portal branches. PVT occurs either in association with cirrhosis or malignancy of liver or may occur without an associated liver disease. Balfour and Stewart (1869) described the first case of PVT in 1868 in a patient with ascites, splenomegaly and variceal dilation. PVT is responsible for $5 \%-10 \%$ of all cases of PH in western countries. Of all cases of $\mathrm{PH}$ in developing countries, $40 \%$ are attributed to PVT (Ogran et al. 2006). Non-cirrhotic prehepatic PH is a consequence of portal vein thrombosis all over the world.

Orllof et al. (2002) determined the site of blood flow obstruction in 200 children and young adults. The authors observed that $67 \%$ of patients showed only portal vein obstruction, whereas $28 \%$ showed portal and splenic vein involvement, and $5 \%$ had portal vein and superior mesenteric thrombosis. Portal vein obstruction can occur at any site of the portal system.

When portal hypertension occurs in the absence of cirrhosis, Extra Hepatic Portal Venous Obstruction (EHPVO) is most often indicated etiologically. Patients with EHPVO are generally young and belong mostly to Asian countries. EHPVO is an important cause of PH among children (Sarin SK and Kapoor D 2002). It is characterized mainly by PVT and is detected with upper gastrointestinal bleeding caused by esophageal 
varices (Poddar et al. 2008). In Japan, 10\%-20\% of variceal bleeds and in the west, $2 \%$ $5 \%$ of variceal bleeds are due to EHPVO (Amitrano et al. 2004). In children, $70 \%$ of all variceal bleeds are due to EHPVO from the Indian subcontinent.

EHPVO can lead to severe bleeding from gastrointestinal varices, ascites, thrombocytopenia from hypersplenism, jaundice and other intrinsic liver diseases (Gibson et al. 1965). Decreased growth velocity and short stature is the complication of EHPVO and after shunt surgery, a medication of EHPVO, there is also declining hepatotropic growth factors and growth spurts usually observed (Kato T et al. 2000). Growth retardation has been seen in $51 \%$ of children in the authors' group of 500 patients due to EHPVO whereas other studies showed this association with decreased levels of IGF-1 and IGF BP-3. Also many kinds of literature explicate a hypothesis that the low supply of blood to the liver due to the formation of systemic collaterals in PVT patients may cause hepatotropic hormone deprivation, thus interfering with the child's global growth (Sarin SK and Agarwal SR 2002).

Computational Fluid Dynamics (CFD) has shown a great potential in computational biology and biomechanics lately due to the limitations of traditional measurement methods. Since CFD uses numerical methods and algorithms to solve and analyze problems that involve fluid flows so its applicability can be exploited for solving blood flow problems. This method can render relatively accurate results of the flow system.

In the study of portal system hemodynamic, Botara et al. (2010) implemented the Reynolds Stress model to describe hemodynamic in portal vein system. He used actual patient imaging of the portal vein in CFD modeling to obtain hemodynamic quantities such as velocities and wall shear stress. A very good correlation between simulation results and clinical data has been obtained by this study.

Petkova et al. (2003) examined blood flow in an idealized model of the portal vein with and without thrombosis using CFD with both a Newtonian and a Non-Newtonian blood viscosity model. The main focus of this study is that to evaluate the impact of thrombosis on the flow behavior.

Steenkiste et al. (2010) carried out a quantitative analysis of the Wall Shear Stress in portal vein in casts of different animal models of portal hypertension and cirrhosis using computational fluid dynamics. This study showed that vascular casting has an important role not only in the morphological evaluation of animal models of portal hypertension and cirrhosis, but also in defining the biological response of the portal vein wall to hemodynamic changes.

George et al. (2008) employed an idealized portal vein model to simulate how the blood coming from the splenic vein (SV) and superior mesenteric vein (SMV) 
distributions between the left and right lobes of the liver. B. Chen et al. (2015) conducted in vitro experimentation and CFD simulation for both an idealized model and specific models based on the CT images acquired from four healthy subjects and two patients with liver cirrhosis. The analysis showed that right-lobe atrophy is significantly influenced by the distribution of blood from the SV. Moreover, the patients with liver cirrhosis had a significantly larger mass fraction of spleen-derived blood in the left portal vein branch (LPV) than healthy subjects, a result consistent with right-lobe atrophy and left-lobe hypertrophy.

It is reported that portal hypertension is one of the major complication in different liver disorders. The obstruction of blood flow in portal vein causes portal hypertension. The objective of the present study is to investigate the change of hemodynamic for the $40 \%$ block and $85 \%$ block in the portal vein even when the flow conditions are identified as a normal portal vein. A gradual reduction of blood pressure as well as the increment of blood velocity and wall shear stress is observed near the block region of the $40 \%$ block portal vein and $85 \%$ block portal vein cases respectively. According to this perspective we get a favorable scenario of portal hypertension for $40 \%$ blocked and $85 \%$ blocked portal vein cases.

\section{MATERIALS AND METHODS}

\section{Problem Statement}

Here we consider Extra Hepatic Portal Vein Obstruction (EHPVO) by forming the different size of the partial block in the main portal vein (without involving intrahepatic obstruction) to evaluate the hemodynamic quantities. We have used a simple 2D geometry according to Petkova et al. (2003) shown in Fig.1, Fig.2 and Fig.3 which has four branches with different flow rates.
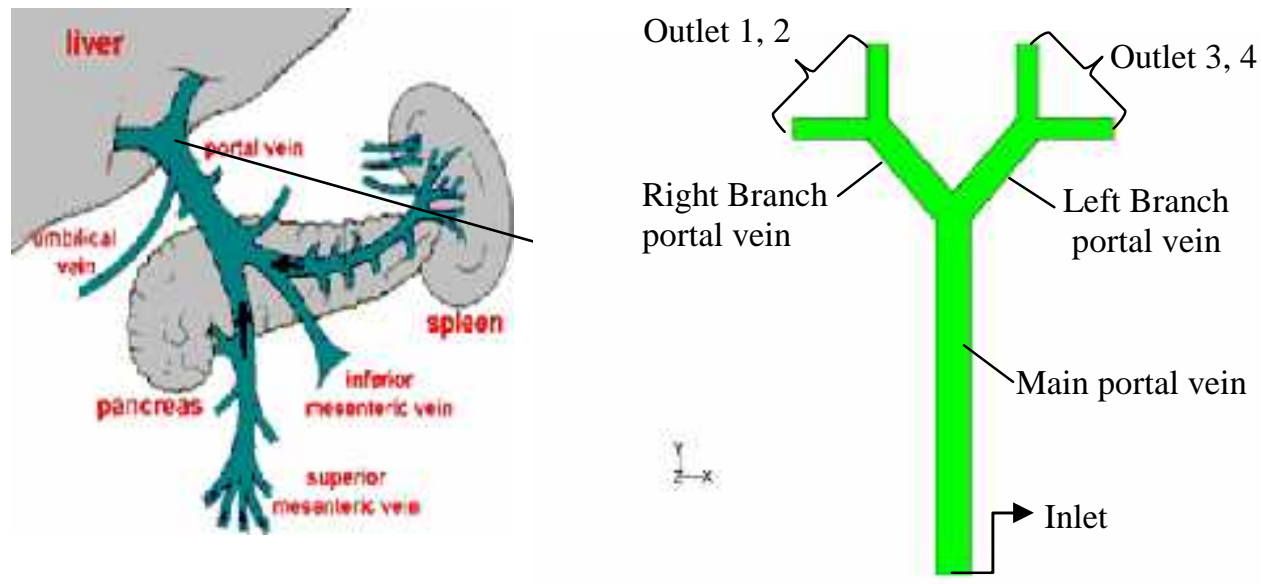

Fig. 1. Portal vein with four branches 


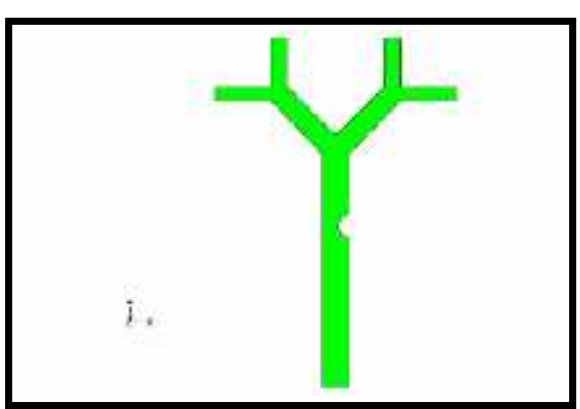

Fig.2: $40 \%$ partially blocked portal vein

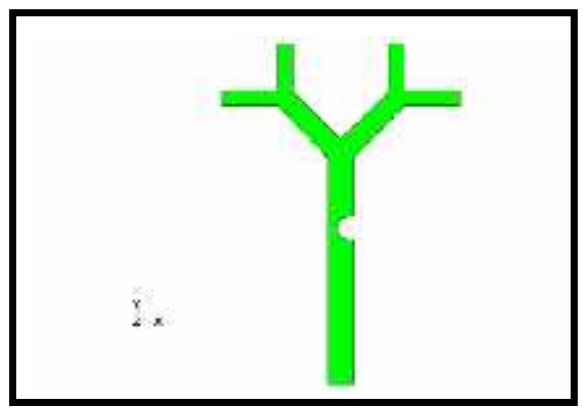

Fig.3: $85 \%$ partially blocked portal vein

Table 1 shows the dimensions of the geometry used in this model.

Table 1: Model dimensions.

\begin{tabular}{ll}
\hline Description & Dimensions $(\mathrm{mm})$ \\
\hline Inlet diameter & $10 \mathrm{~mm}$ \\
First branching diameter & $8 \mathrm{~mm}$ \\
Outlet diameter & $6 \mathrm{~mm}$ \\
Total height & $150 \mathrm{~mm}$ \\
\hline
\end{tabular}

Fig. 1 is the normal portal vein where Fig. 2 and Fig. 3 have $40 \%$ block and $85 \%$ block in the main portal vein. The model geometry was constructed by using Gambit 2.2.30 a component of the fluent package. The meshing scheme is composed of elements and type. The elements selected were quadrilateral defines the shape of the element used to mesh and the type parameter defines the meshing algorithm which in our case was paved. After the geometry is successfully meshes, the zone types are specified. A mesh file is exported for use in Fluent 17.2.

\section{GOVERNING EQUATIONS}

Blood in the Portal vein manifests laminar flow with a Reynolds number ranging from 663 to 730 . Because the Portal vein is far from the heart, the influence of the cardiac cycle can be disregarded. In our study, the $2 \mathrm{D}$ blood flow is assumed to be steady and laminar. The three basic laws of conservation of mass, momentum and energy are solved for incompressible flows of non-Newtonian fluid.

The 2D continuity equation or equation for conservation of mass for incompressible flow with steady state can be written as:

$$
\operatorname{div} \vec{V}=0
$$

Where $\vec{V}$ is the velocity field.

The Navier-Stokes equation for the incompressible flow with steady state in vector form is described as the following form : 


$$
\rho(\overrightarrow{\mathrm{V}} \cdot \nabla) \vec{V}=-\nabla P+\nabla \cdot(\vec{\tau})+\rho \vec{g}
$$

Where, $\rho$ is the fluid density, $\vec{V}$ is the velocity field, $P$ is the static pressure, $\bar{\tau}$ is the stress tensor and $\rho \bar{g}$ is the gravitational body force. Though the veins are narrow but the density of blood is very high so there is some gravitational effect which is taken into consideration for this model (Petkova et al. 2003, S.M. George 2008, Yin et al 2001).

The stress tensor $\bar{\tau}$ is given by

$$
\bar{\tau}=\mu\left[\left(\nabla \bar{V}+\nabla \bar{V}^{T}\right)-\frac{2}{3} \nabla \cdot \overline{V I}\right]
$$

Where $\mu$ is the molecular viscosity, I is the unite tensor and the second term of the right hand side is the volumetric dilation.

For incompressible Newtonian fluids viscosity $\mu$ is independent of the rate of deformation. But for the non-Newtonian fluids $\mu$ is a function of the rate of deformation. Thus for some non-Newtonian fluids, the shear stress can be written in terms of a nonNewtonian viscosity $\eta$ :

$$
\bar{\tau}=\eta(\bar{D}) \bar{D}
$$

where $\bar{\tau}$ is the stress tensor and $\bar{D}$ is the rate of deformation tensor defined by

$$
\bar{D}=\left(\frac{\partial u_{j}}{\partial x_{i}}+\frac{\partial u_{i}}{\partial x_{j}}\right)
$$

The Energy equation is

$$
\operatorname{\rho div}(i \bar{V})=-P \operatorname{div} \bar{V}+\operatorname{div}) \kappa \operatorname{grad} T)
$$

where $i$ is the specific internal energy, $\bar{V}$ is the velocity field, $\kappa$ is the thermal conductivity and $\mathrm{T}$ is the temperature of the fluid. In the case of incompressible flow there is no need to establish the linkage between the energy equation and the equations of continuity and momentum.

In our model the non-Newtonian power law model has been used and the nonNewtonian viscosity $\eta$ is considered to be a function of the shear rate $\dot{\gamma}$ only. $\dot{\gamma}$ is related to the second invariant of $\bar{D}$ and defined as

$$
\dot{\gamma}=\sqrt{\bar{D}: \bar{D}}
$$

For our model, the non-Newtonian viscosity is calculated by using the following formula (FLUENT 6.0 Manual, Chapter 7.3.5)

$$
\eta=K y^{n-1} e^{\frac{T_{0}}{T}}
$$

where $K$ is the power law consistency factor, $\mathrm{n}$ is the flow behavior index, $T_{0}$ is the 
reference temperature. It may be noted that if $\mathrm{n}=1$ then fluid corresponds to Newtonian behavior, if $\mathrm{n}<1$ then fluid corresponds to shear-thinning or pseuwwdo-plastics behavior and if $\mathrm{n}>1$ then shear-thickening or dilatants behavior.

\section{BASIC ASSUMPTIONS}

The portal vein or hepatic portal vein is a blood vessel that carries blood from the gastrointestinal tract and spleen to the liver. The blood from the portal vein supplies $80 \%$ of the liver's blood and contains nutrients that were absorbed from the digestive tract. As the heart pulsates blood at a certain frequency, the blood that flows from that organ has a pulsatile behavior. However as the blood flows to the rest of the body and the blood vessels get thinner, the blood flow assumes a steady state behavior. In a mathematical point of view two parameters are used to know whether the flow is closer to steady or pulsatile behavior. Those parameters are Reynolds number (Re) and the Womersely number $(\alpha)$. The following equations are used to determine the value of $\operatorname{Re}$ and $\alpha$

$$
\begin{array}{r}
\operatorname{Re}=\frac{\rho V D}{\mu} \\
\text { and } \quad \alpha \frac{D}{2} \sqrt{\frac{\rho \omega}{\mu}}
\end{array}
$$

where, $\rho$ is the blood density, $\bar{V}$ is the mean velocity, D is the diameter of the blood vessel, $\omega$ is the angular frequency, $\omega=2 \pi \times 0.5 \mathrm{rad} / \mathrm{s}$ and $\mu$ is the blood viscosity. In our model, the Womersely number is $\alpha=5.26$ according to equation (10). Since $\alpha<10$, flow can be assumed steady. Reynolds number is $\operatorname{Re}=708$ according to equation (9) so the flow is laminar.

Blood viscosity can be defined as the quantity that describes the blood's resistance to flow, which is being deformed by either shear stress or tensile stress. For different parts of the circulatory system, different behaviors of blood viscosity seem to happen. In healthy straight large blood vessels, where the blood flow is in high strain rate blood shows Newtonian behavior, however, in small blood vessels, the strain rate is low and the blood viscosity shows a shear-thinning behavior.

The difference in portal blood flow between normal subjects and patients remains unclear. Kayacetin et al. (2004) and Vyas et al. (2002) found a significant decrease in portal blood flow in patients but Yin et al. (2001) found a significant increase in portal blood flow. Several other studies found no significant difference between the two groups. Portal hypertension is considered present when the portal vein flow velocity is less than $21 \mathrm{~cm} / \mathrm{sec}$ (Haag et al. 1999). 
Since the angles between the branches of the portal vein and their radius do not play any significant impact on the hemodynamic changes so we are not concerned about those.

\section{BOUNDARY CONDITIONS}

In every CFD model, apart from the geometry definition and the consequent mesh, there is the need of prescribing boundary conditions. Blood flows by entering and exiting blood vessels, which have walls that do not allow blood to pass through. Therefore it is clear that for the inlet and outlet as well as for the walls there must be boundary conditions. For the inlet a velocity inlet is used. As for the outlets an outflow condition is used. Rigid wall conditions were assumed according to the literature (Botara et al. 2010) and neglecting the elastic property (Vimmr et al. 2010).

For our study, we consider an average velocity of superior mesenteric vein (SMV) and splenic vein (SV) for the inlet velocity under portal hypertension condition. The operating pressure is 2731.1 Pascal and inlet velocity is $20 \mathrm{~cm} / \mathrm{s}$. Since the left part of the liver is approximately two times smaller than the right part and therefore has a blood flow twice as high (Orloff et al. 2002). All outlets were classified as an outflow in which the flow split was taken with flow rate weighting 0.70 for each outlet 1 and 2 in the right branch and 0.50 for each of outlet 3 and 4 in left branch. Portal vein carries the rich blood of nutrients which has a large amount of water (approximately 70\%). For this reason we consider alike-blood as the working fluid whose physical properties (specific heat and thermal conductivity) are similar to water with density $\rho=1060 \mathrm{~kg} / \mathrm{m}^{3}$. As mentioned previously, blood shows a shear-thinning behavior and is often modeled as a NonNewtonian fluid. This behavior is dependent on the strain rate of the fluid. However, a diseased portal vein with decreased blood flow will show a lower strain rate in the range of $1-200 \mathrm{~s}^{-1}$ where the shear-thinning will be important (Wang et al. 2009).

The flow properties of blood used in this study is given in the following table.

Table 2. Flow properties.

\begin{tabular}{ll}
\hline Power law index $(\mathrm{n})$ & 0.4851 \\
\hline Consistency index $\kappa\left(\mathrm{kg}-\mathrm{s}^{\mathrm{n}-2} / \mathrm{m}\right)$ & 0.2073 \\
\hline Reference temperature $\left({ }^{0} \mathrm{~K}\right)$ & 310 \\
\hline Minimum viscosity limit $\eta_{\max }(\mathrm{kg} / \mathrm{m}-\mathrm{s})$ & 0.00125 \\
\hline Maximum viscosity limit $\eta_{\min }(\mathrm{kg} / \mathrm{m}-\mathrm{s})$ & 0.003 \\
\hline
\end{tabular}

\section{NUMERICAL METHOD}

The pressure based solver was chosen due to the modeling of low-speed incompressible flow. FLUENT solves the governing equations for mass and momentum 
in order to obtain the velocity field. To do this a control volume based technique is used. The domain is divided into discrete control volumes based on the computational grid. The governing equations are integrated over the individual control volumes to construct algebraic equations. The discretized equations are linearized and solved to yield values for unknown variables. The formulation for the solver was implicit. The velocity formulation was absolute and porous formulation was superficial velocity. The GreenGauss Cell based gradient option was used. For the pressure-velocity coupling the SIMPLE (Semi-Implicit Method for Pressure-Linked Equations) algorithm was selected which uses the relationship between velocity and pressure correction to enforce mass conservation and obtain the pressure field. The computations are considered to be converged when the residues for continuity, momentum and energy are less than $10^{-5}$.

\section{VALIDITY AND VERIFICATION}

To validate our model we simulated blood flow through a normal portal vein (without block) with the boundary conditions as was done by Petkova (2008). We compared velocity distribution with that of Petkova at $y=20 \mathrm{~mm}$. As displayed in Fig. 4, the results mostly agreed with Petkova model results except for minor differences which are due to 3D geometry used by Petkova. We proceed with the assumption that our model is a valid model.

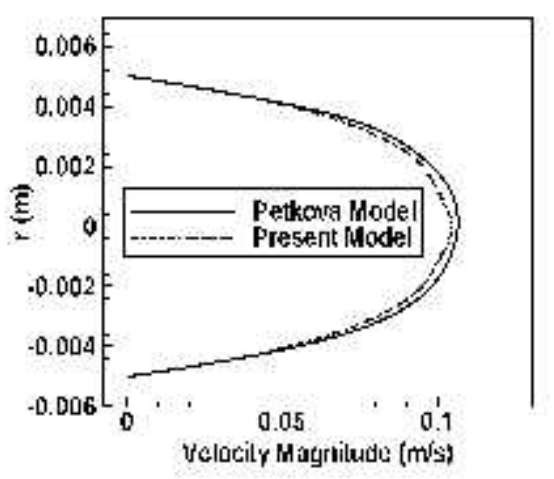

Fig. 4. The velocity distribution at $y=20 \mathrm{~mm}$

\section{RESULTS AND DISCUSSION}

In this section presents simulation results of blood flow in the normal portal vein as well as $40 \%$ block and $85 \%$ block in the main portal vein. The velocity was uniform at the inlet and was set $20 \mathrm{~cm} / \mathrm{s}$. We compared velocity distribution of blood flow through the normal portal vein at three different positions, along with the streamwise direction, 
which are at $\mathrm{y}=50 \mathrm{~mm}, \mathrm{y}=63 \mathrm{~mm}$ and $\mathrm{y}=75 \mathrm{~mm}$ respectively. The velocity distributions are the same as expected for all the three positions which are displayed in the Fig.5.

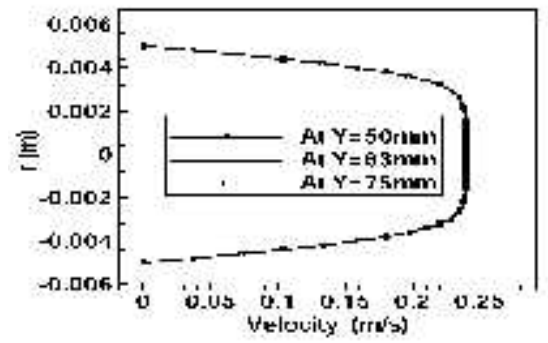

Fig. 5. Velocity profiles for normal portal vein

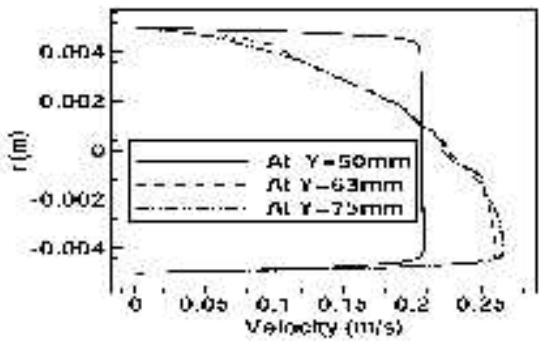

Fig. 6. Velocity profiles for $40 \%$ partial blocked in the portal vein

The comparisons of velocity profile for $40 \%$ block and $85 \%$ block in the portal vein in the same region as mentioned above are represented by Fig.6 and Fig.7. With the comparison of Fig.5 as

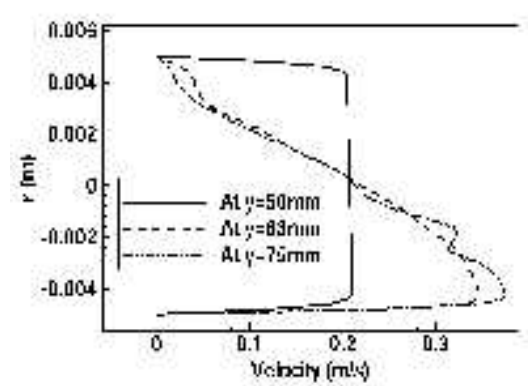

Fig. 7. Velocity profiles for $85 \%$ partial blocked in the portal vein

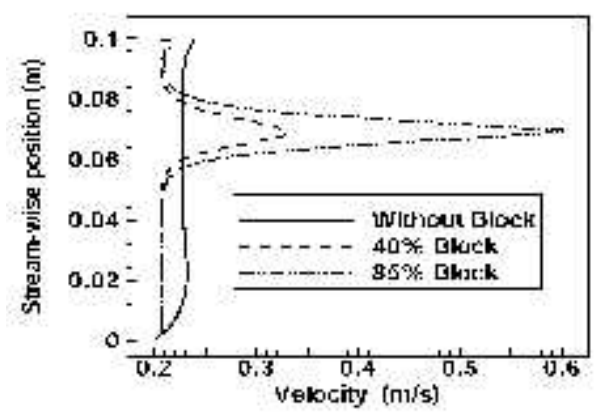

Fig. 8. Velocity profiles along the line at $r=$ $-1 \mathrm{~mm}$ for the three cases

Fig. 8. represents the velocity profile along the line in the position at $\mathrm{r}=-1 \mathrm{~mm}$ in the main portal vein for the three cases. This figure depicts the scenario of onward velocity distribution beside the region where the block has been created. The average velocity in the main portal vein are $20 \mathrm{~cm} / \mathrm{s}$ for the normal portal vein, $22.19 \mathrm{~cm} / \mathrm{s}$ for the $40 \%$ blocked portal vein and $24.95 \mathrm{~cm} / \mathrm{s}$ for the $85 \%$ blocked portal vein respectively. There is a high blood velocity through the $40 \%$ blocked portal vein and is a much higher blood velocity through the $85 \%$ blocked portal vein compared with the normal portal vein in consequence of stuffing flow through the narrow flow path. 


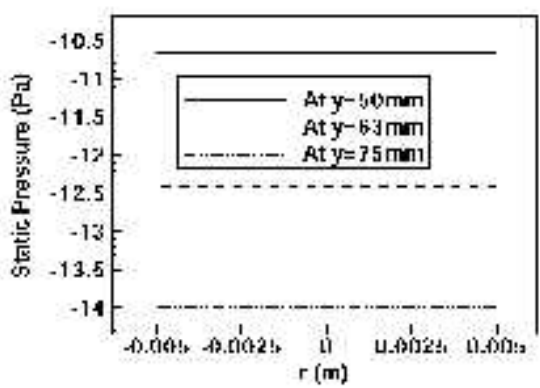

Fig. 9. Pressure distribution for normal portal vein

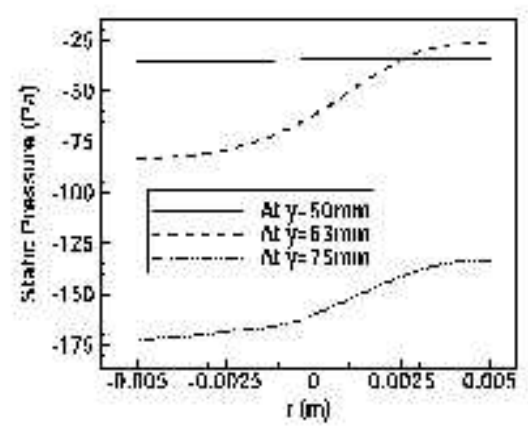

Fig. 11. Pressure distribution for $85 \%$ block portal vein

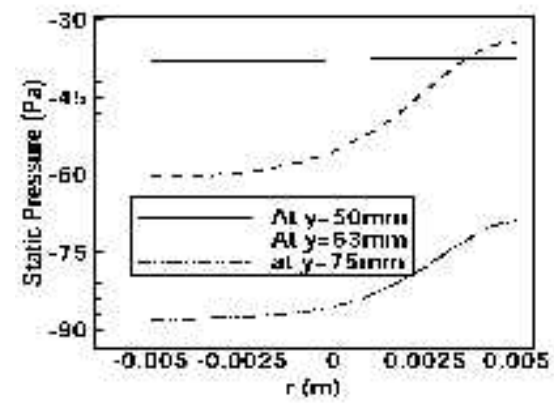

Fig. 10. Pressure distribution for $40 \%$ block portal vein

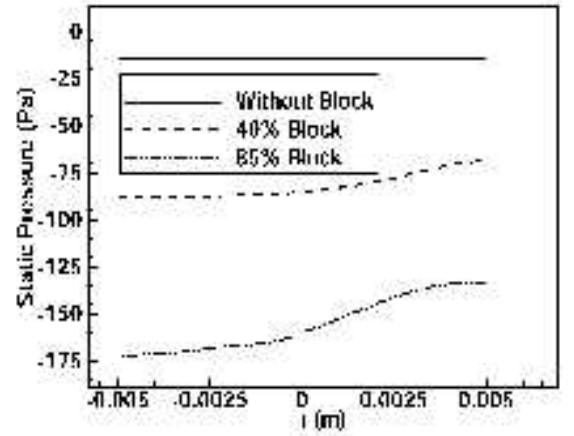

Fig. 12. Comparison of static pressure distribution after the block region for the three cases

Fig. 9, Fig. 10 and Fig. 11 show the assimilation of static pressure distribution at three different positions which are at $\mathrm{y}=50 \mathrm{~mm}, \mathrm{y}=63 \mathrm{~mm}$ and $\mathrm{y}=75 \mathrm{~mm}$ for normal portal vein, $40 \%$ blocked and $85 \%$ blocked portal vein successively. Pressure distribution at these regions, in Fig.9, does not vary significantly for normal portal vein. But a noticeable variation of pressure distribution, in Fig.10 and Fig.11, is observed for $40 \%$ blocked and $85 \%$ blocked portal vein. Along the line at $\mathrm{y}=50 \mathrm{~mm}$ gives a stagnant pressure distribution for the three cases because of there is no variation of velocity distribution in that position. But along the line at $\mathrm{y}=63 \mathrm{~mm}$, before the block region, gives an inequitable pressure distribution for the $40 \%$ blocked and $85 \%$ blocked portal vein as shown in Fig.10 and Fig.11. The same visualization has been observed along the line at $\mathrm{y}=75 \mathrm{~mm}$, after the block region. High velocity magnitude at these regions has been responsible for this environment of pressure distributions. This observation has been granted by the statement of Bernoulli equation.

Fig. 12 shows the comparison of static pressure distribution at the region of the block for the three cases respectively. Since near the block region the velocity is very high and 
thereby the pressure is low. This may cause more shrinkage of the vein which stops blood circulation gradually. Its gives the most favorable picture of portal hypertension.

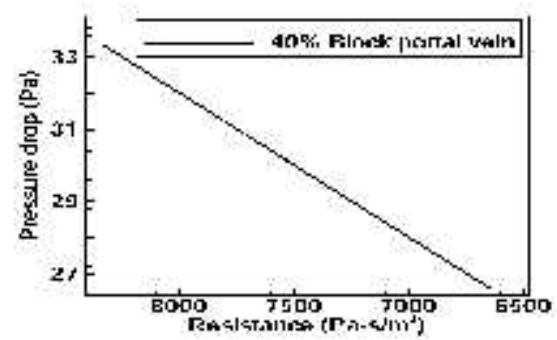

Fig. 13. Resistance of flow in the block region for $40 \%$ block portal vein.

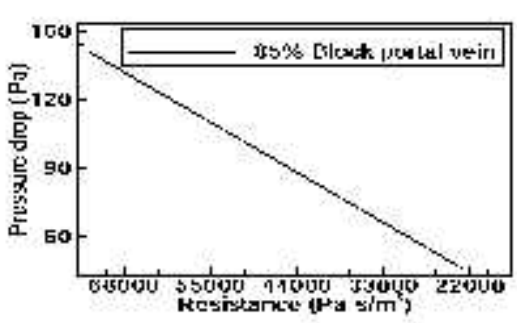

Fig. 14. Resistance of flow in the block region for $85 \%$ block portal vein.

Fig. 13 and Fig. 14 show the resistance of flow near the block region for $40 \%$ blocked and $85 \%$ blocked portal vein. Resistance controls the blood flow rate of the blood vessels. Resistance of blood vessel is calculated by using Ohm's law. This law can be stated as - the higher the resistance, the slower the flow rate. The resistance of flow in case of normal portal vein is very minimal which is neglected here. On the other hand, the resistance of flow in case of $85 \%$ blocked portal vein is higher than that of $40 \%$ blocked portal vein. This is due to the larger amount of reduction of cross-sectional area in $85 \%$ blocked portal vein compared to $40 \%$ block portal vein. In small width of the vessels, it is hard to push a lot of blood through it. It implies increased the pressure difference and decreased flow rate through the vessel.

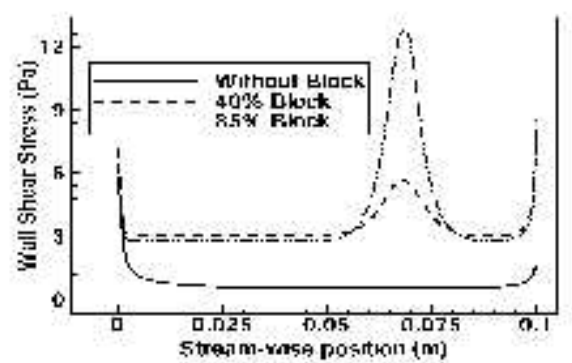

Fig. 15. Shear stress on the wall in the main portal vein for three cases

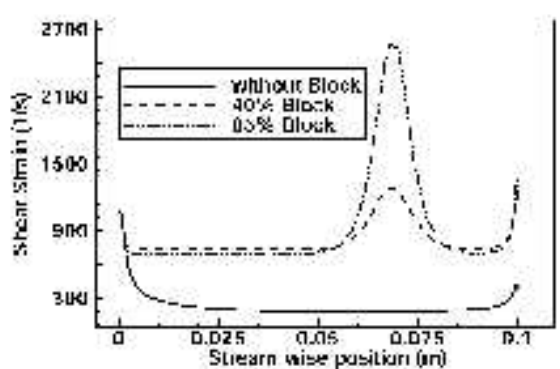

Fig. 16. Shear strain on the wall in the main portal vein for three cases

Fig. 15 is the combined stage of shear stress on the wall for the normal portal vein as well as $40 \%$ blocked and $85 \%$ blocked portal vein respectively. Also a collection of shear strain on the wall from the three cases is shown by Fig. 16. It can be seen that the wall shear stress and the wall shear strain is almost constant in the flow path for the normal portal vein but a distinguishable depictures for the remainder two cases. The blockage in the main portal vein decreases the available cross section area. This reduction of available 
cross section area introduces higher strain rates and shear stress for $40 \%$ blocked and $85 \%$ blocked portal vein. With an increase of strain rate may contribute to change of viscoelasticity property of blood.

Under the normal circumstances, blood flow through the portal vein is split between the right branch of the portal vein (RPV) and left branch of the portal vein (LPV) in such way that the conservation of mass flow through the liver is maintained. Since the right lobe of the liver is twice than left lobe of the liver so the RPV carries much blood than LPV. The average velocity for RPV and LPV are $16.02 \mathrm{~cm} / \mathrm{s}$ and $10.13 \mathrm{~cm} / \mathrm{s}$ respectively for the normal portal vein. The average velocity for RPV and LPV are $15.95 \mathrm{~cm} / \mathrm{s}$ and $11.38 \mathrm{~cm} / \mathrm{s}$ is observed in the $40 \%$ blocked portal vein case. Moreover the average velocities for RPV and LPV in the $85 \%$ blocked portal vein case are $15.58 \mathrm{~cm} / \mathrm{s}$ and $11.46 \mathrm{~cm} / \mathrm{s}$.

Table 3 gives the flow rate of RPV and LPV for the normal portal vein, $40 \%$ blocked portal vein and $85 \%$ blocked portal vein. It is noted that, the flow rate through the RPV

Table 3. The RPV and LPV Flow rate

\begin{tabular}{|l|c|c|}
\hline \multirow{2}{*}{} & \multicolumn{2}{|c|}{ Flow rate $\left(\mathrm{m}^{3} / \mathrm{s}\right)$} \\
\cline { 2 - 3 } & RPV & LPV \\
\hline Normal portal vein & 0.00121748 & 0.000782621 \\
\hline $40 \%$ Block portal vein & 0.00121721 & -0.000782531 \\
\hline $85 \%$ Block portal vein & 0.00121735 & -0.00078258 \\
\hline
\end{tabular}

and LPV for normal portal vein case, $40 \%$ blocked and $85 \%$ blocked portal vein cases is almost constant. The constancy is due to the maintenance of conservation of mass through the portal vein. But the flow rate in LPV contains a minus sign for $40 \%$ blocked and $85 \%$ blocked portal vein cases. This minus sign indicates a decrease of pressure in the direction of flow through the LPV. The decreases of blood pressure through LPV may cause atrophy of the left lobe of liver.

To get the effect of irregular blood flow to the liver, this model is designed with different sizes of block in the portal vein under healthy liver condition and results are presented by considering block sizes effects. This kind of investigation is rare in the literature. The nearest one which is similar to that of ours in some respects is done by Petkova et al. (2003) where they considered not a healthy liver but a liver affected by liver cirrhosis. 
A vortex flow near the blocked region for the $40 \%$ blocked and $85 \%$ blocked portal vein is pointed out by Fig. 17 and Fig. 18.
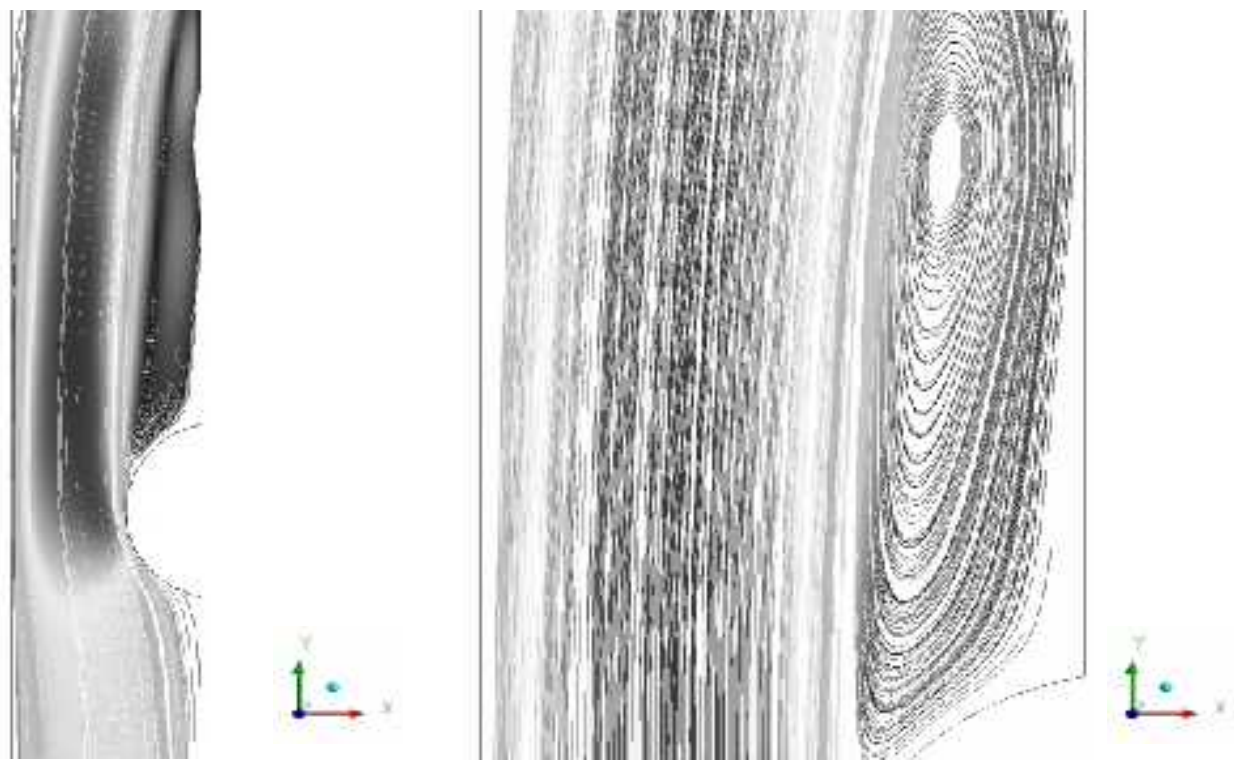

Fig. 17: Streamlines (a) with magnified view of vortex flow (b) for $40 \%$ blocked portal vein

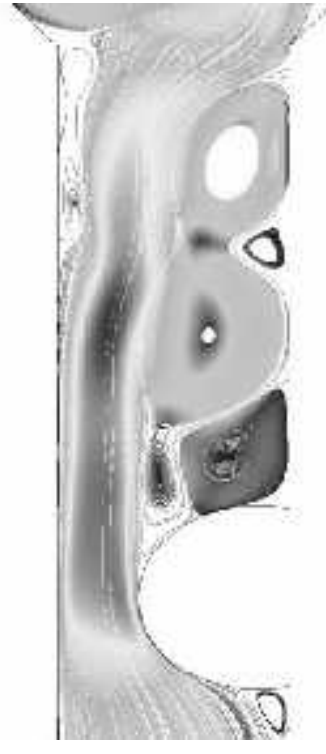

(a)

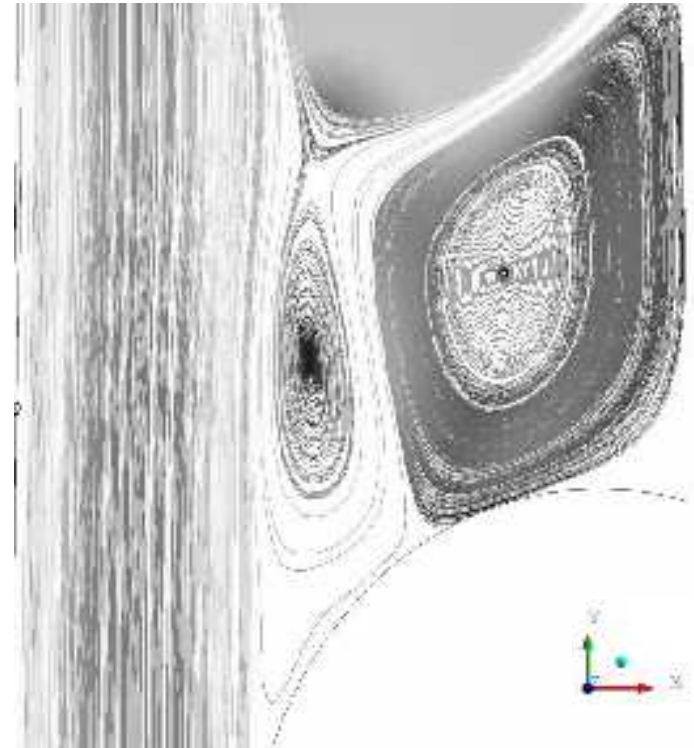

(b)

Fig. 18. Streamlines (a) with magnified view of vortex flow (b) for $85 \%$ blocked portal vein 
Fig.19 and Fig.20 represent the reverse flow through the RPV and LPV of the portal vein. This vortex and the reverse flows are an indication of advance portal hypertension stage. It can be said that corrosion is possible to happen in such locations. This may leads to form thrombosis in the main portal vein, RPV and LPV of the portal vein.

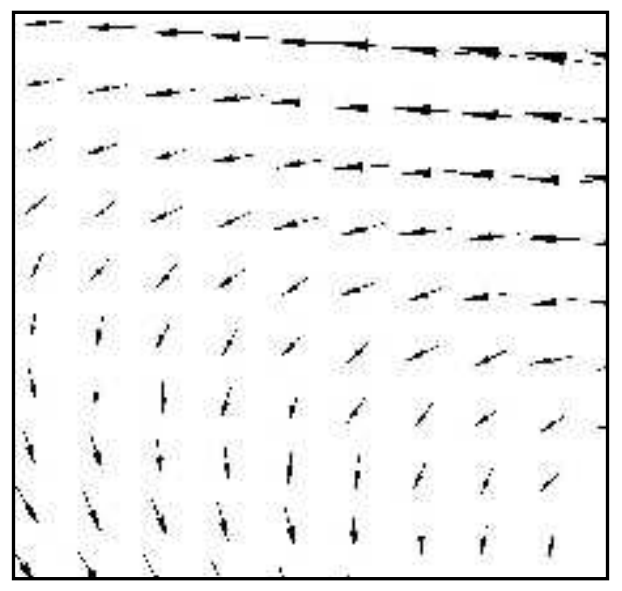

Fig.19. Reversed flow through RPV

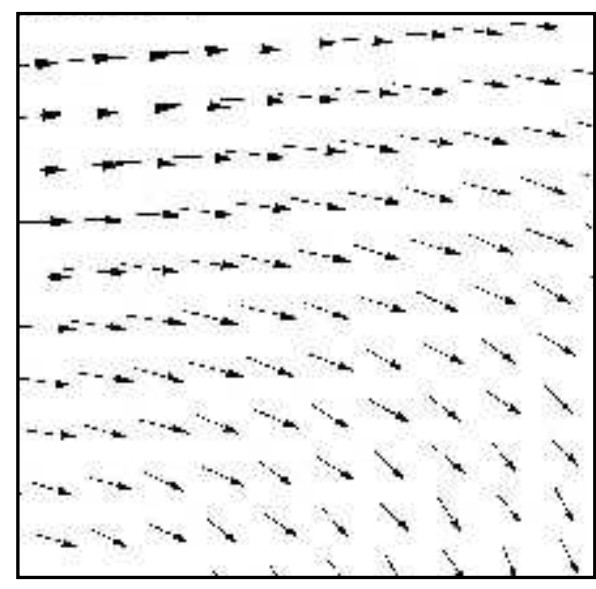

Fig. 20. Reversed flow through LPV

\section{CONCLUSION}

The simulated results of blood flow through portal vein models confirmed that the presence of block in the main portal vein have an effect on the variations of hemodynamic behavior. It is observed that there is a back flow through the blocked portal vein which may haphazard the systemic circulation and can be responsible for the enlargement of spleen and varicose veins in the stomach. Moreover the effect of blockage leads to produce the possibility of forming new thrombosis in the left and right branches of the portal vein which may cause the Hypertrophy and Atrophy complex to the liver. Thus the liver function and its architecture may be affected by the obstructions.

\section{REFERENCES}

Amitrano, L, Guardascione1 MA, Brancaccio V, Margaglione M, Manguso F, Iannaccone L, et al. 2004. Risk factors and clinical presentation of portal vein thrombosis in patients with liver cirrhosis. J Hepatol.40: 736-41

Balfour, G.W. and Stewart T.G. 1869. Cases of enlarged spleen complicated with ascites, both depending upon varicose dilatation and thrombosis of the portal vein. Edinb Med J, 14: 589598.

Bari, K, Garcia-Tsao G., 2012, Treatment of portal hypertension. World J Gastroenterol., 18(11): 1166-75.

Botara, C.C., T. Vasileb, S. Strangeub, S. Clichicic, P. S. Agachia, R. Badeab, P. Mircead and M. V.Cristeaa 2010. Validation of CFD simulation results in case of portal vein blood flow. $20^{\text {th }}$ European symposium on computer Aided process Engineering ESCAPE 20.

BritishLiverTrust British Liver Trust, http://www.britishlivertrust.org.uk/home.aspx. 
Chen, B., X.Li, X. K. Wang, Y. S. Pu, Z. F. Li, P. Nie and K. Su 2015. Computational hemodynamics of portal vein hypertension in hepatic cirrhosis patients. Bio-Medical Materials and Engineering. 26: 233-243.

Christophe Van Steenkiste, Bram Trachet, Christophe Casteleyn, Denis van Loo, Luc Van Hoorebeke, Patrick Segers, Anja Geerts, Hans Van Vlierberghe and Isabelle Colle.2010. Vascular corrosion casting: analyzing wall shear stress in the portal vein and vascular abnormalities in portal hypertensive and cirrhotic rodents: Laboratory Investigation. 90: 15581572 .

Denk, H. 2004., Pathology of portal hypertension. Journal of Gastroenterology and Hepatology. 19: $\mathrm{S} 346-\mathrm{S} 348$.

FLUENT, 2006, www.fluent.com.

George, S.M. 2008. Hemodynamic investigation of the liver using magnetic resonance imaging and computational fluid dynamics, Ph.D. Dissertation, Georgia Institute of Technology.

Gibson, JB, Johnston GW, Fulton TT, Rodgers HW. 1965. Extrahepatic Portal-Venous Obstruction. Br J Surg. 52: 129-39.

Haag, K. Rossle, M. Ochs, A. Huber, M. Siegerstetter, V. Olschewski, M. Berger, E. Lu, S C \& Blum.1999. Correlation of duplex sonography findings and portal pressure in 375 patients with portal hypertension. American Journal of Roentgenology. 172(3):631-635.

Kato, T., Romero R, Koutouby R, Mittal NK, Thompson JF, Schleien CL, et al. 2000. Portosystemic shunting in children during the era of endoscopic therapy: Improved postoperative growth parameters, J PediatrGastroenterolNutr. 30: 419-25.

Kayacetin, E., Efe, D., and Dogan, C.2004. Portal and splenic hemodynamics in cirrhotic patients: relationship between esophageal variceal bleeding and the severity of hepatic failure. Journal of Gastroenterology. 39(7): 661-667.

Ogren, M, Bergqvist D, Wahlander K, Eriksson H, Sternby NH., 2006, Trousseau's syndrome-what is the evidence? A population-based autopsy study. Thromb Haemost. 95: 541-545.

Orloff, MJ, Orloff MS, Girard B, Orloff SL. 2002. Bleeding esophagogastric varices from extrahepatic portal hypertension: 40 years' experience with portal-systemic shunt. J Am Coll Surg. 194: 717-728.

Petkova, A. Hossain, J. Naser, and E. Palombo. 2003. CFD modeling of blood flow in portal vein hypertension with and without thrombosis. Third International Conference on CFD in the minerals and Process Industries. 527-530.

Petkova, S. 2008. Investigation of portal vein blood flow in cirrhotic portal hypertension using computer-base and physical modelling methods. PhD thesis, Swinburne University of Technology.

Poddar, U., Thapa B.R., Rao K.L. and Singh K. 2008. Etiological spectrum of esophageal varices due to portal hypertension in Indian children: is it different from the West? $J$ GastroenterolHepatol. 23: 1354-1357.

Sarin, SK., Agarwal SR 2002. Extrahepatic portal vein obstruction, Semin Liver Dis, 22: 43-58.

Sarin, SK., Kapoor D, 2002. Non-cirrhotic portal fibrosis: Current concepts and management. $J$ Gastroenterol Hepatol. 17: 526-34.

Sarin, SK., Sollano JD, Chawla YK, Amarapurkar D, Hamid S, Hashizume M et al.2006. Consensus on extra-hepatic portal vein obstruction. Liver Int. 26: 512-9.

Sherlock and Dooley, 2002, Disease of the Liver and Biliary System. Blackwell Science.

Sherlock. 1974. Classification and Functional Aspects of Portal Hypertension. The American Journal of Surgery. 127: 121-128. 
Vimmr, J. and A. Jonásová. 2010. Non-Newtonian effects of blood flow in complete coronary and femoral bypasses. Mathematics and Computers in Simulation. 80: 1324-1336.

Vyas, K., Gala, B., Sawant, P., Das, H., Kulhalli, P., and Mahajan, S.2002. Assessment of portal hemodynamics by ultrasound color doppler velocimetry in liver cirrhosis, Indian Journal of Gastroenterology. 21(5): 176-178.

Wang, D. and J. Bernsdorf. 2009. Lattice Boltzmann simulation of steady Non-Newtonian blood flow in a 3D genericstenosis case. Computers and Mathematics with Applications. 58: 10301034.

Yin, X., Lu, M., Huang, J., Xie, X., and Liang, L. 2001. Color doppler velocity profile assessment of portal hemodynamics in cirrhotic patients with portal hypertension: correlation with esophageal variceal bleeding. Journal of Clinical Ultrasound, 29(1): 7-13.

(Received revised manuscript on 5 November, 2017) 